\title{
L'EDUCAZIONE INTERCULTURALE: STIMOLO PER UNA CITTADINANZA EUROPEA E DEMOCRATICA ${ }^{[1]}$
}

\author{
Elvi Piršl \\ Marina Diković \\ Università Juraj Dobrila di Pola \\ Dipartimento di studi in lingua italiana
}

\section{Riassunto}

In questo lavoro, entro l'ambito dell'interculturalità, si vuole offrire una panoramica delle finalità dell'insegnamento e dell'educazione ad una cittadinanza europea, un quadro della competenza interculturale di base necessaria alla formazione dei futuri cittadini europei, come pure una breve sintesi delle esperienze croate avviate in questi campi. Educare all'interculturalità significa, innanzitutto, acquisire e comprenderne i concetti chiave, rispettare i principi dell'interculturalità, capire le conseguenze della discriminazione, dei pregiudizi e degli stereotipi, nonché permettere agli alunni l'applicazione attiva del sapere, delle abilità e della competenza interculturale, oltre a quelle generali, indispensabili in una società democratica e culturalmente pluralistica. Leducazione alla cittadinanza europea si sviluppa nel corso dell'intera esistenza del singolo individuo e si manifesta in tutte le sue sfere (lifelong e lifewide learning) attraverso la realizzazione di un'educazione formale, non formale ed informale. Il dialogo interculturale è un fattore indispensabile e di estrema importanza per una cittadinanza europea in una società fondata sui valori.

Parole chiave: educazione interculturale, educazione alla cittadinanza europea, competenza interculturale

\section{Introduzione}

La diversità culturale non è solo oggetto di comprensione, tolleranza e solidarietà, ma è anche una fonte di arricchimento della propria persona e della propria cultura e una grande occasione di comprensione dei limiti del sistema culturale di appartenenza.

Nessuna discriminazione nellerogazione del curricolo scolastico dovrebbe essere compiuta per motivi riguardanti il sesso, la razza, letnia, la religione, la lingua, le opinioni politiche, le condizioni psicofisiche, sociali ed economiche. Nella concreta prassi d'insegnamento, le prestazioni degli insegnanti non sono uniformi, ma tengono conto

[1] Questo saggio nasce come parte del progetto di ricerca: Educazione per la competenza interculturale (303-3031173-0983) approvato dal Ministero della scienza, dell'educazione e dello sport della Repubblica di Croazia. 
delle diverse condizioni personali, sociali e culturali degli alunni, al fine di conseguire i risultati desiderati. In ottemperanza a quanto detto, si dovrà tendere alla valorizzazione di tutte le risorse umane, professionali, culturali, materiali e tecnologiche disponibili in ogni realtà scolastica. Siccome il concetto di uguaglianza delle opportunità implica il principio che gli allievi, per poter raggiungere il massimo delle loro possibilità, data la loro diversità culturale e sociale, devono essere seguiti con modalità adeguate alle loro reali capacità. Dunque, nella scuola bisogna applicare strategie di insegnamento flessibili, che usino una base comune a tutti, ma che poi siano differenziate per alcuni casi particolari, casi che in una classe possono essere più o meno numerosi. Il problema di fondo è che la diversità non diventi disuguaglianza delle possibilità di crescita e di apprendimento. Con ladozione del principio di uguaglianza, le diversità, indiscutibili delle epoche storiche precedenti, sono state percepite come disuguaglianze o come differenze funzionali (Messeri, in Fabbri e Rossi, 2007, p. 27). Il concetto di disuguaglianza, spiega l'autore, esprime delle asimmetrie di posizione rispetto a un parametro discriminante, mentre la differenza funzionale indica un'articolazione razionale di posizioni, intese come un insieme di attività ascritte a un ruolo in un sistema organizzato. Le disuguaglianze sono un carattere strutturale di tutte le forme di società e rappresentano uno degli oggetti costitutivi dell'analisi sociologica (Messeri, in Fabbri e Rossi, 2007, p. 28).

In una società globale e multiculturale, il diritto di cittadinanza assume il significato di rispetto e dovrebbe riferirsi ad ogni individuo in relazione ai suoi diritti fondamentali. L'educazione alla cittadinanza europea si rivolge ad una società in rapida evoluzione e globalizzazione che investe tutto il pianeta. A questo punto sono inevitabili alcune domande: Quali sono gli obiettivi affidati all'educazione alla cittadinanza europea? Quali sono le competenze necessarie per la formazione di questi cittadini? Qual è il ruolo degli insegnanti e della scuola (delluniversità) nella formazione dei futuri cittadini europei?

\section{L'obiettivo dell'educazione alla cittadinanza europea e democratica}

La cittadinanza, nel senso più ampio del termine, indica un diritto e anche una responsabilità di partecipazione, insieme agli altri, alla vita sociale ed economica e agli affari pubblici della comunità (Libro bianco sul dialogo interculturale, 2008, p. 29). La cittadinanza rappresenta un elemento indispensabile per il dialogo interculturale. Si può dire che leducazione alla cittadinanza democratica sia molto importante per il funzionamento di una società libera, tollerante, aperta, inclusiva per la coesione sociale, la comprensione reciproca, la solidarietà, il dialogo interculturale e religioso e la parità fra donne e uomini (Libro bianco sul dialogo interculturale, 2008, p. 30).

Leducazione alla cittadinanza europea comincia nella scuola, ma coinvolge l'apprendimento durante tutto l'arco della vita e in tutti gli ambiti della vita di una persona (lifelong e lifewide learning). Quest'ambito specifico dell'educazione alla cittadinanza europea si può sviluppare e realizzare sia nelleducazione formale sia nelleducazione non-formale o informale ${ }^{[2]}$. I concetti chiave dell'educazione alla cittadinanza europea

\footnotetext{
[2] Tenendo fede alla letteratura più recente, l’educazione interculturale non viene realizzata soltanto attraverso una istruzione formale (che è quella scolastica) o non formale (che comprende corsi ed iniziative nate fuori della scuola), ma anche attraverso i canali ancora poco conosciuti delleducazione informale.
} 
sono i valori della cittadinanza, la qualità e la partecipazione alla vita democratica di una comunità, come pure la promozione della sua cultura e dei suoi valori.

Leducazione alla cittadinanza europea comprende sia l'educazione civica, storica, politica e l'educazione ai diritti umani, sia l'attenzione al contesto mondiale delle società nonché il patrimonio culturale. Inoltre, favorisce gli approcci pluridisciplinari e l'apprendimento delle conoscenze, competenze e comportamenti.

\section{Ripensare ai valori}

Educare alla cittadinanza europea e democratica significa, anzitutto, "alfabetizzare" (cfr.: Sarsini, 2003) al linguaggio specifico per comprendere e promuovere la cultura della democrazia e i suoi valori, vale a dire a possedere e ridefinire i concetti ed i principi di fondo che sono alla base di parole come libertà, uguaglianza, giustizia, partecipazione, pluralismo, rispetto reciproco, responsabilità, sviluppo sostenibile. Si tratta di un linguaggio che pone un accento sullo studio sistematico non soltanto del testo costituzionale, che per Sarsini è quello italiano, ma anche della Dichiarazione universale dei diritti umani (1948), indispensabile per educare al senso della cittadinanza europea. In questi documenti si trovano concetti chiave riguardo i valori-bisogni e dirittidoveri che consentono a tutti noi, non solo di comprendere, ma anche di contribuire a trasformare la realtà sociale nella direzione e nella realizzazione di un mondo più civile, umano e ragionevole. Tutti i valori elencati, di fronte alle grandi novità odierne, dalla crisi economica mondiale, alle guerre di popolo nei paesi arabi, alla fuga dall'Africa di numeri consistenti di persone, devono essere ripensati.

D’altronde, l'educazione alla cittadinanza europea e democratica attribuisce un'importante rilevanza alle dimensioni valoriali dell'insegnamento e della vita di relazione vissuta all'interno della scuola e dell'università ${ }^{[3]}$ come: il rispetto reciproco, la partecipazione, la collaborazione, l'impegno competente e responsabile, la cooperazione e la solidarietà. Dunque, si deve evidenziare che educare alla cittadinanza europea e democratica significa educare ad una partecipazione attiva nelle comunità di appartenenza e ad una padronanza di conoscenze, atteggiamenti e sentimenti da parte degli individui che renda efficace la loro azione di cittadini. In una tale prospettiva e dal momento che il fenomeno della multiculturalità è irreversibile, l'educazione ad una cittadinanza europea, comporta anche uneducazione alla diversità culturale ed alla comprensione reciproca.

Alcuni autori distinguono i valori civici nei quali includono gli obiettivi e le idee (ad esempio: onestà, dignità) dagli orientamenti valoriali, come ad esempio liberalismo o conservatorismo (cfr.: Piršl, Vican, 2004). Il rapporto tra i diritti e gli interessi della comunità deve essere costantemente nutrito soprattutto di sviluppo dei valori civici. Ogni cittadino che vive in una comunità democratica dovrebbe praticare dei valori civici. I cittadini devono essere educati a questi valori sin dalla loro infanzia, nei loro contesti di vita familiare, scolastica e sociale. Essi si sviluppano nelle relazioni quotidiane, durante le varie attività a scuola, in famiglia, nella comunità e in altri contesti. Si tratta di

[3] La dimensione valoriale dell'insegnamento deve essere praticata dalla scuola elementare fino all'università. 
competenze che aiutano non soltanto a risolvere i problemi nel modo migliore all'interno delle comunità e nelle relazioni quotidiane interpersonali, ma permettono di diventare cittadini consapevoli, autocritici e flessibili.

\section{Educare all'interculturalità}

Si ha un nuovo periodo nello sviluppo dell'educazione interculturale nei primi mesi degli anni ' 80 , quando, a causa del ricongiungimento familiare e un alto tasso di natalità tra le comunità degli immigrati, si rinforzano le caratteristiche multietniche e multiculturali delle società europee occidentali. Da allora, quest'approccio è in continua evoluzione, ele strategie, i metodi e le forme d'insegnamento dell'educazione interculturale iniziano a descrivere nel modo migliore la situazione attuale (cfr.: Perotti, 1995). Alcuni autori (cfr.: Desinan, 1997) hanno evidenziato che oggi, nel sistema scolastico, l'educazione interculturale non si riferisce più solo ad una categoria di alunni (i figli degli immigranti) e ai loro bisogni specifici, ma alla necessità di adattare l'insegnamento alle nuove esigenze imposte dal contesto pluriculturale della società cioè di tenere conto, nella pedagogia globale, della situazione multilingue e pluriculturale. Occorre pertanto che il compito degli educatori sia indirizzato a favorire un'informazione critica e una formazione di base dei giovani e dei soggetti in età evolutiva che sarà tanto più profonda e radicale se esperienze significative e confronti produttivi si svilupperanno proprio negli anni del maggior imprinting e della maturazione consapevole, onde evitare deviazioni e stereotipi (Biagioli, 2005, p. 28).

Secondo alcuni autori (cfr.: Spajić-Vrkaš e vari, 2004) educare all'interculturalità significa: (1) apprendere i concetti fondamentali nel campo della cultura e delle relazioni interculturali, (2) comprendere le caratteristiche della propria cultura e la diversità culturale nel mondo, (3) rispettare i principi dell'interculturalità, (4) sviluppare un dialogo aperto e un'identità interculturale ${ }^{[4]}$ in conformità con le esigenze della comunità culturalmente pluralistica e democratica, compresa la sensibilità culturale e la competenza linguistica, (5) rendersi conto delle conseguenze della discriminazione, dei pregiudizi e degli stereotipi nel confronto con l'Altro, come pure dell'importanza della tolleranza e della solidarietà, (6) dare la possibilità agli alunni/studenti di acquisire conoscenze, abilità - competenze richieste per un'attiva partecipazione nella società democratica e culturalmente pluralistica (7), costruire la consapevolezza sui diritti e sulle responsabilità, sulle norme dei valori e degli atteggiamenti come pure sulle questioni etiche e morali di una comunità. L'accento, quindi, non dovrebbe essere posto solo sull'apprendimento e sulla conoscenza della propria cultura, quanto sulla comprensione dei principi di base necessari per il funzionamento di una comunità culturalmente pluralistica, come la responsabilità civica, la solidarietà, la consapevolezza critica dei fattori etnici, razziali, religiosi e altri che provocano la disuguaglianza, la discriminazione, il conflitto. Anche i più recenti documenti riguardanti l'ambito dell'interculturalità indicano alcuni orientamenti

[4] Il concetto di identità coinvolge la funzione delle relazioni tra le persone mentre una trasformazione più significativa del processo e dello sviluppo dell'identità interculturale (open identity) è il cambiamento della comprensione dosservazione del mondo intorno a noi. Identità interculturale significa accettazione degli elementi nuovi e di quelli già esistenti, auto comprensione, auto accettazione ed auto confidenza più ampie, come pure una creatività più profonda che ci permetta di affrontare con maggiore facilità le nuove sfide. 
sul dialogo interculturale ed evidenziano tutti i principi sopra elencati. Il Libro bianco sul dialogo interculturale (2008, p. 25), ad esempio, promuove una politica d'azione fondata sul dialogo interculturale che dipende dalla (1) governance democratica della diversità culturale, passa attraverso (2) la partecipazione e la cittadinanza democratica, richiede (3) l'acquisizione di competenze culturali, necessità (4) spazi di dialogo aperti e infine deve essere condotta su (5) scala internazionale.

Gli studiosi evidenziano diverse definizioni per quanto riguarda l'educazione interculturale. Quindi essa non va vista soltanto come una soluzione al problema della presenza sempre maggiore di alunni culturalmente diversi nelle scuole (cfr.: Previšić, Mijatović, 2001), non si esaurisce nei problemi posti dalla presenza di alunni stranieri a scuola, ma si estende alla complessità del confronto tra culture in una dimensione europea e mondiale dell'insegnamento e costituisce la risposta più alta e globale al razzismo e all'antisemitismo (Biagioli, 2005, p. 27). Sotto il cappello del Consiglio Europeo la scuola ha assunto in Europa un approccio prevalentemente interculturale, anziché multiculturale, di tipo americano, anche se con aspetti organizzativi e pratici significativamente diversi da Paese a Paese. Le origini dell'educazione interculturale fondano le proprie radici nei primi arrivi di alunni stranieri e nella loro integrazione nel sistema scolastico dei Paesi d'accoglienza. Una tale fase è però completamente superata e oramai è diventato necessario avviarsi verso traguardi più elevati.

\section{Competenza interculturale - competenza essenziale nell'ambito della cittadinanza europea e democratica}

Una cittadinanza europea ed una educazione interculturale adeguata richiedono che le conoscenze vengano applicate nell'operatività e diventino competenza. Che cosa si intende per competenza? La competenza rappresenta la combinazione dei saperi, delle abilità, degli atteggiamenti e delle caratteristiche personali che permettono all'individuo di partecipare attivamente ed efficacemente nelle specifiche situazioni (scolastiche, universitarie). La competenza coinvolge le componenti: cognitive, affettive e comportamentali di ciascuna personalità soggettiva. Il concetto di competenza è definito nei vari testi specifici secondo diverse accezioni di significato come: risorsa cognitiva necessaria per gli output professionali; set di comportamenti organizzativi per una prestazione; capacità e comportamenti organizzativi e professionali posseduti o da sviluppare e premiare; fattore di sviluppo delle imprese; caratteristiche intrinseche del business e delle persone che fanno parte di unorganizzazione. Una tale definizione mette in evidenza soprattutto la parte professionale e imprenditoriale della competenza. È però presente, in letteratura, una diversa concezione della competenza, che proviene dalle scienze sociali. Secondo questa versione, la competenza rappresenta lo spazio d'interpretazione in cui, a livello individuale e collettivo, vengono esercitate esperienza, cognizione (sapere) e volontà (attitudini) (cfr.: Viteritti, 2005). Ed è questo secondo concetto di competenza che va collocato alla base della competenza interculturale.

Oltre a ciò, come primo gradino, per la competenza interculturale non vanno trascurate quelle che sono state chiamate "competenze-chiave". Si tratta di un complesso di 
competenze di base, essenziali, che consentono agli individui di partecipare attivamente in diversi contesti sociali, così da affrontare i continui sviluppi e trasformazioni della società. Il nuovo cittadino europeo deve continuare ad apprendere durante tutta la vita, aggiornare costantemente conoscenze e abilità, imparare ad adattarsi in modo flessibile a un mondo in rapido mutamento, caratterizzato da forte interconnessione (cfr.: Tosolini e vari, 2007). Come si vede si tratta di competenze che sono indispensabili sia per i soggetti autoctoni sia per i soggetti stranieri che entrano a far parte della comunità. Servono a ciascuno di loro, ma servono anche a facilitare i rapporti interculturali e sociali.

Secondo la Raccomandazione del Parlamento Europeo e del Consiglio del 18 dicembre 2006, relativa alle competenze chiave per un apprendimento permanente, le competenze chiave sono:

a) imparare ad imparare - intesa come abilità del soggetto di perseverare e organizzare il proprio apprendimento a livello individuale e di gruppo;

b) competenze sociali e civiche - sono le competenzepersonali, interpersonali ed interculturali che consentono a una persona di partecipare in modo efficace $e$ costruttivo alla vita sociale e lavorativa;

c) il senso di iniziativa e di imprenditorialità - visto come capacità di una persona di tradurre le idee in azioni e attività attraverso un processo di negoziazione efficace.

Queste tre competenze richiedono la capacità di: conoscere se stessi, il proprio carattere, i propri bisogni e desideri, nonché i propri punti deboli e forti (autocoscienza). Richiedono, inoltre, la capacità di esprimersi sia a livello verbale che non verbale (comunicazione efficace), di comprendere ed ascoltare gli altri, soprattutto quando si tratta di diversità etniche, religiose e culturali (empatia), di saper affrontare, decidere e risolvere in modo consapevole e costruttivo le più diverse situazioni e problemi di vita.

La competenza interculturale diventa allora la capacità di aumentare progressivamente la possibilità di comprendere la realtà e l'esperienza della differenza in maniera sempre più complessa. Molti studiosi pensano che per avere una competenza interculturale occorra sapere quanto possibile su gruppi etnici diversi, in modo da generare quella che gli americani hanno chiamato "mente aperta" (open mind), vale a dire tollerante e disposta alla comprensione su quanti più gruppi etnici al mondo, padroneggiare bene molte lingue, avere chiari riferimenti valoriali legati a usanze e comportamenti. Le competenze principali relative al contatto fra culture identificate dai ricercatori sono raggruppabili intorno a dieci dimensioni principali: abilità comunicative, tolleranza dell'ambiguità, empatia, apertura mentale, flessibilità, capacità di sapersi concentrare sia sul compito sia sulle relazioni, atteggiamento positivo riguardo allapprendimento, tolleranza nei confronti di stili e culture diverse, conoscenze di tipo culturale, capacità di avere successo in ambienti diversi.

Nell'ambito della competenza interculturale un ruolo di primo piano è occupato dalla competenza comunicativa. Su una tale competenza avevano già scritto, in precedenza, tra altri, Spitzberg e Cupach (1984), i quali, in un loro studio, avevano messo in evidenza l'importanza che nel sistema complesso delle relazioni sociali assumono sia la capacità di comunicare, e cioè di inviare e di 
mettere in comune dei messaggi, sia quella di ascoltare e capire i messaggi ricevuti. Bennett (1993) sostiene che lo sviluppo della competenza interculturale può essere presentato come un modello di maggiore o minore sensibilità interculturale corrispondente a sei fasi della crescita personale (rifiuto, difesa, minimizzazione, accettazione, adattamento, integrazione). Questo modello di sviluppo presuppone un continuo miglioramento nel confronto con le differenze culturali, spostandosi dall'etnocentrismo, attraverso le fasi di maggiore riconoscimento e accettazione delle differenze, all'etnorelativismo.

Per la competenza interculturale Byram (1997) intende il rapporto fra la conoscenza delle altre culture (le pratiche culturali) e le abilità di analisi dell'interazione interculturale, il ruolo della comunicazione non verbale, lampiezza del concetto di competenza interculturale, l'attenzione per i tratti psicologici e la capacità di agire, l'influenza dei fattori sociali e politici nel definire e valutare la competenza. Il quadro di riferimento proposto da Byram (1997) si articola in cinque ambiti, due relativi alle conoscenze, due alle abilità e uno agli atteggiamenti: conoscenza degli altri, dei processi sociali e dei gruppi sociali (savoirs); conoscenza di sé, consapevolezza culturale critica, compresa l'abilità del saper riconoscere e valutare pratiche e prodotti della propria e di altre culture (savoir séngager); abilità relative all'interpretazione e alla relazione (savoir comprendre), abilità relative alla scoperta e/o all'interazione (savoir apprendre/faire), atteggiamenti che permettono di relativizzare se stessi (valori, credenze e comportamenti a partire da prospettive più ampie) e di valorizzare credenze, comportamenti e valori degli altri (savoir être).

Nell'insegnamento ai fini di una competenza interculturale è molto importante sia la comunicazione verbale e non verbale sia i processi verbali e non verbali (cfr.: Piršl, 2005). I processi verbali non includono solo il modo in cui le persone parlano tra loro, ma anche le attività cognitive, come il pensiero e la comprensione del significato di alcune parole usate durante la conversazione. I processi non verbali trasmettono degli atteggiamenti anche emotivi nei confronti dellinformazione. Pertanto, una comunicazione di successo significa pure "saper osservare" anche se l'osservazione non è strettamente inerente agli atteggiamenti emotivi che entrano a far parte della comunicazione verbale.

L'insegnamento della competenza interculturale è essenziale per una cultura democratica e la coesione sociale. Le diverse organizzazioni sociali dovrebbero essere un esempio dello sviluppo del dialogo interculturale e delleducazione inclusiva a tutti i livelli, ma dare anche possibilità agli alunni di mostrare la qualità delle relazioni interculturali nell'ambito scolastico. 


\section{La grande sfida dell'educazione nella formazione dei futuri cittadini europei}

La cittadinanza può essere definita come un insieme di diritti e di obblighi (cfr.: Bryan S. Turner, 1993, in Perczynski e Vink, 2002). Rappresenta le attività desiderabili che sono in funzione della partecipazione nella comunità (cfr.: Kymlicka e Norman, 1995, in Perczynski e Vink, 2002) come pure le attività riguardanti i diritti delle minoranze. Kymlicka (2003) nel suo libro parla della cittadinanza multiculturale e ritiene che il cittadino fuori dalla cultura nazionale non possa avere libertà di scelta. Il concetto di cittadinanza nei documenti del Consiglio d'Europa si riferisce alla cittadinanza democratica basata sui principi e sui valori del pluralismo, sul primato della legge, sul rispetto della dignità umana e della diversità culturale. Nei documenti dell'Unione Europea alla cittadinanza attiva si determina che il cittadino europeo deve possedere il sapere, le abilità e con le competenze necessarie deve partecipare nellaccettazione delle decisioni che devono essere prese nella sfera della propria comunità.

Il sistema scolastico è sicuramente rilevante, dato che molti giovani trascorrono in queste istituzioni parecchi anni in qualità di utenti. La scuola dovrebbe avere il compito di gettare le basi di questo processo in quanto progetta, organizza e attiva percorsi per favorire lo sviluppo del pensiero critico, dell'autonomia personale e delle capacità decisionali. Seguendo questa direzione, è chiaro che l'educazione e l'insegnamento non sono rivolti solo ad alcuni soggetti in alcuni momenti particolari della vita, bensì rappresentano un processo che coinvolge tutti per agevolare un percorso di crescita che consenta di affrontare le scelte e la partecipazione attiva alla vita sociale.

Lobiettivo principale della scuola è di formare e potenziare le capacità dei suoi utenti di conoscere se stessi, l'ambiente in cui vivono, $i$ mutamenti culturali e socioeconomici, le offerte formative affinché possano essere protagonisti di un personale progetto di vita individuale e sociale.

Il ruolo degli insegnanti e dei docenti è di determinare i fini dell'educazione alla cittadinanza europea attiva. Nell'ambito scolastico (universitario) la formazione degli alunni/studenti permette di acquisire e/o perfezionare nuove metodologie didattiche e condizioni di apprendimento favorevoli a promuovere competenze che siano alla base non solo delleducazione alla cittadinanza europea, democratica e attiva, ma anche dei diritti umani, dell'interculturalità e dell'educazione alla pace.

\section{Educazione all'interculturalità e alla cittadinanza europea e democratica - esperienza della Croazia}

L'ambito dell'educazione alla cittadinanza è l'idea guida per la costruzione e la responsabilizzazione degli alunni come cittadini consapevoli, attivi e responsabili in tutte le politiche e pratiche educative d'Europa, come pure nelle politiche e pratiche dei Paesi in transizione (cfr.: Diković, 2010).

L'educazione all'interculturalità e l'educazione alla cittadinanza democratica (europea), come materie o corsi di studio, non sono sistematicamente e del tutto 
inserite nel sistema scolastico della Croazia. Malgrado i documenti strategici rivelino l'importanza della promozione del pluralismo culturale nelleducazione, l'inserimento di questi principi è stato ostacolato da numerosi fattori concettuali e organizzativi.

In Croazia nel 1999 è stato ideato il Programma Nazionale di educazione ai Diritti umani e alla cittadinanza democratica (1999). Il Programma d'insegnamento per la scuola elementare (2006) ha introdotto questi contenuti nell'ambito della gestione integrata dei contenuti educativi, ossia in quello interdisciplinare, come materia facoltativa, attraverso attività extracurriculari, sotto forma di progetti e anche attraverso il curricolo scolastico. Il documento normativo sulleducazione più recente, il Curricolo nazionale per l'educazione prescolare e per la scuola dellobbligo e la scuola superiore (2010) prevede l'applicazione dei contenuti dell'educazione alla cittadinanza e delleducazione all'interculturalità come argomento interdisciplinare o come tema integrato in campo socio-umanistico. Questo documento è basato su una cittadinanza responsabile, su un attivo ed efficace svolgimento della coscienza democratica civica degli alunni e su uno sviluppo dell'identità, dell'apprendimento e del rispetto per gli altri, nella convinzione che i grandi problemi mondiali possono essere risolti solo sulla base dei principi della democrazia. Lo scopo ultimo è di istruire ed educare gli alunni e i giovani ad una cittadinanza con tutte le qualità, in modo che possano essere in grado di partecipare attivamente in una società democratica.

Dato che non è obbligatorio inserire il Programma Nazionale di educazione ai Diritti umani e alla cittadinanza democratica (1999) nei programmi delle scuole, la promozione dei principi e valori democratici e interculturali dipende in gran parte dalla buona volontà degli insegnanti, dalle loro competenze, dalla sensibilità e motivazione, come pure dal sostegno dalle autorità scolastiche.

A questo grande compito, del tutto nuovo, di formare i giovani ad una cittadinanza europea, ma anche, parallelamente, all'accettazione ed al rispetto della diversità culturale, come componente fondamentale per la cittadinanza europea, è chiamata non solo la scuola di ogni ordine e grado, ma anche l'università. A questo riguardo è doveroso constatare che ci sono pochissimi esempi nei programmi universitari di inserimento dell'educazione alla cittadinanza democratica europea e delleducazione all'interculturalità come materie obbligatorie o opzionali. Eventualmente vengono svolte come parte integrante di alcuni contenuti di altre materie (pedagogia, sociologia, antropologia, ecc.). L'Università di Pola si è già posta il problema e da alcuni anni gli studenti del Dipartimento di scienze della formazione frequentano il corso Educazione ai diritti umani come obbligatorio, gli studenti del Dipartimento di scienze umanistiche frequentano i corsi Educazione ai diritti umani e alla cittadinanza democratica, Competenza e comunicazione interculturale e Pedagogia interculturale come opzionali, mentre invece gli studenti del Dipartimento di studi in lingua italiana frequentano il corso opzionale del curricolo educativo Competenza e comunicazione interculturale. 


\section{Conclusione}

Cittadini si è per diritto, ma lo si diventa anche per educazione e per compartecipazione alla cultura comunitaria. A questo scopo è necessaria l'acquisizione di conoscenze relative alle idee, valori, tecniche adeguate alla convivenza civile del proprio tempo, del proprio paese e del mondo contemporaneo. L'educazione interculturale non è più una strategia educativa diretta al solo scolaro straniero, ma diventa una forma dell'educazione generale ed una componente essenziale per una cittadinanza europea e democratica.

In Croazia nel Piano e programma d'insegnamento per la scuola elementare (2006) l'educazione alla cittadinanza e l'educazione all'interculturalità sono inserite come parte integrante dei contenuti educativo-istruttivi, e nel Curricolo nazionale per l'educazione prescolare e per la scuola dellobbligo e la scuola superiore (2010) come tema interdisciplinare. Nella politica e nella pratica della maggior parte dei Paesi europei e di quelli in transizione, leducazione alla cittadinanza è entrata come esplicito obiettivo ed approccio educativo che si realizza in una specifica materia d'insegnamento.

Educandolegenerazioni attuali alla presa di coscienza delle proprie responsabilità e agli altri valori, i canoni delleducazione alla cittadinanza europea democratica e all'interculturalità devono garantire anche in futuro il mantenimento e la salvaguardia delle società democratiche e multiculturali e promuovere la partecipazione attiva di tutti gli individui alla vita politica, civile e culturale.

\section{BIBLIOGRAFIA}

BENNETT, M. J., 1993, Education for intercultural experience, Me: Intercultural Press, Yarmouth.

BIAGIOLI, R., 2005, Educare all'interculturalità. Teorie, modelli, esperienze scolastiche, Franco Angeli, Milano.

BYRAM, M., 1997, Teaching and assessing intercultural communicative competence, Multilingual Matters, Clevedon.

Curricolo nazionale per l'educazione prescolare e per la scuola dellobbligo e la scuola superiore, 2010, Ministero della scienza, dell'educazione e dello sport della Repubblica di Croazia, Zagabria.

DESINAN, C., 1997, Orientamenti di educazione interculturale, Franco Angeli, Milano.

Dichiarazione universale dei diritti umani, 1948, Nazioni Unite.

DIKOVIĆ, M., 2010, Hrvaška izobraževalna politika v kontekstu vzgoje in izobraževanja za državljanstvo, in Sodobna pedagogika, 3, 192-207.

FABBRI, L., ROSSI, B., (a cura di), 2007, La costruzione della competenza interculturale. Agire educativo e formazione degli insegnanti, Guerini scientifica, Milano. KYMLICKA, W., 2003, Multikulturalno građanstvo. Liberalna teorija manjinskih prava, Naklada Jasenski i Turk, Zagreb. 
Libro bianco sul dialogo interculturale. „Vivere insieme in pari dignità‘, 2008, Consiglio d'Europa, Strasburgo.

PERCZYNSKI, P., VINK, M., 2002, Citizenship and Democracy: A Journey to Europe's Past, in Citizenship Studies, 6, 2, 183 -199.

PEROTTI, A., 1995, Pledoaje za interkulturalni odgoj i obrazovanje. Educa, Zagreb.

Piano e programma d'insegnamento per la scuola elementare, 2006, Ministero della scienza, dell'educazione e dello sport della Repubblica di Croazia, Zagabria.

PIRŠL, E., VICAN, D., 2004, Europske demokratske vrijednosti i regionalizam, in Pedagogijska istraživanja, 1, 1, 89-102.

PIRŠL, E., 2005, Verbalna i neverbalna interkulturalna komunikacija, in BENJAK, M., POŽGAJ-HADŽI, V. (a cura di), Bez predrasuda i stereotipa, Izdavački centar Rijeka, Rijeka, 50-90.

PREVIŠIĆ, V., MIJATOVIĆ, A., (a cura di), 2001, Mladi u multikulturalnom svijetu. Stavovi srednjoškolaca u Hrvatskoj. Interkultura, Zagreb.

Programma Nazionale delleducazione per $i$ Diritti umani e la cittadinanza democratica, 1999, Governo della Repubblica di Croazia.

Raccomandazione del Parlamento Europeo e del Consiglio, 2006, Unione europea, Brusseles.

SARSINI, D., 2003, Il corpo in Occidente. Pratiche pedagogiche, Carocci editore, Roma.

SPAJIĆ-VRKAŠ, V., STRIČEVIĆ, I., MALEŠ, D., MATIJEVIĆ, M., 2004, Poučavati prava i slobode: Priručnik za učitelje osnovne škole s vježbama za razrednu nastavu, Istraživačko-obrazovni Centar za ljudska prava i demokratsko građanstvo Filozofskog fakulteta u Zagrebu, Zagreb.

SPITZBERG, B. H., CUPACH, W. R., 1984, Interpersonal communication competence, CA: Sage, Beverly Hills.

TOSOLINI, A., GIUSTI, S., POPPONI MORELLI, G., 2007, A scuola di intercultura. Cittadinanza, partecipazione e interazione. Edizioni Erickson, Trento.

VITERITTI, A., 2005, Identità e competenze. Soggettività e professionalità nella vita contemporanea, Guerini, Milano.

\section{INTERKULTURALNI ODGOJ I OBRAZOVANJE: POTICAJ ZA EUROPSKO I DEMOKRATSKO GRAĐANSTVO}

U radu se, u sklopu područja interkulturalizma, daje pregled svrhe nastavei odgoja te obrazovanja za europsko građanstvo, okvir temeljne interkulturalne kompetencije potrebne u oblikovanju budućih građana Europe, kao i kraći sažetak hrvatskoga iskustva iz ovoga područja. Poučavati u području interkulturalizma znači, prije svega, usvojiti i razumjeti ključne pojmove, poštivati principe interkulturalnosti, shvatiti posljedice diskriminacije, predrasuda i stereotipa te omogućiti učenicima aktivnu primjenu potrebnih znanja, vještina i interkulturalne kompetencije, uz ostale, u demokratskim i kulturno pluralnim društvima. Odgoj i obrazovanje za europsko građanstvo odvija se tijekom čitavoga života pojedinca i u svim njegovim životnim područjima (lifelong 
e lifewide learning), a realizira se $\mathrm{u}$ formalnom, neformalnom i informalnom odgoju i obrazovanju. Interkulturalni je dijalog neophodan čimbenik građanstva i značajan je za funkcioniranje društva temeljenoga na vrijednostima.

Ključne riječi: interkulturalni odgoj i obrazovanje, odgoj i obrazovanje za europsko građanstvo, interkulturalna kompetencija

\section{INTERCULTURAL EDUCATION: INCENTIVE FOR EUROPEAN AND DEMOCRATIC CITIZENSHIP}

This paper, within the area of interculturalism, seeks to provide an overview of teaching and education for European citizenship, a frame of the basic intercultural competence necessary for formation of future European citizens, as well as a short summary of Croatian experience in this area. Teaching interculturalism primarily means acquiring and understanding the key concepts, respecting the principles of interculturalism, understanding the consequences of discrimination, prejudices and stereotypes, and also enabling students to actively apply the necessary knowledge, skills and intercultural competence, beside others, in democratic and culturally plural societies. Education for European citizenship is a lifelong learning in any aspect of individual life (lifelong e lifewide learning), realised as formal, non-formal and informal education. Intercultural dialogue is a required factor of citizenship, so important for the functioning of society based on values.

Key words: intercultural education, education for European citizenship, intercultural competence 